Published in final edited form as:

ACS Appl Mater Interfaces. 2016 September 21; 8(37): 24361-24367. doi:10.1021/acsami.6b05534.

\title{
Maximizing the Supported Bilayer Phenomenon: Liposomes Comprised Exclusively of PEGylated Phospholipids for Enhanced Systemic and Lymphatic Delivery
}

\author{
Matthew T. Haynes and Leaf Huang* \\ The Center for Nanotechnology in Drug Delivery, Division of Molecular Pharmaceutics, Eshelman \\ School of Pharmacy, The University of North Carolina at Chapel Hill, Chapel Hill, North Carolina \\ 27599, United States
}

\begin{abstract}
Traditional liposomes degrade into lower-order micelles when PEGylated to even minor degrees (6-7 mol \%) and therefore can offer only limited steric exclusion against opsonization during in vivo delivery. In this work, we present for the first time a liposome coated exclusively by PEGylated phospholipids, utilizing lipid-coated calcium phosphate $(\mathrm{CaP})$ cores of diverse sizes $(10-15 \mathrm{~nm}, 30-40 \mathrm{~nm})$ as well as varying polyethylene glycol (PEG) chain lengths (350-5000 Da). Such fully-PEGylated liposome calcium phosphate (LCP) particles exhibit a PEG chain length-dependent circulation longevity and robust immune evasion, while facilitating both strong accumulation within solid tumors upon intravenous injection and a more rapid and extensive lymphatic drainage upon subcutaneous administration. Further, these fully-PEGylated liposomes remain amenable to active targeting strategies which facilitate improved degrees of focused distribution and nanoparticle uptake, represent a lipid packing density commensurate with the formation of a lipid bilayer, and avoid use of scale-limited physical resuspension methods. We expect such improved delivery properties to translate into improved therapeutic safety and efficacy for a variety of systemic and lymphatic diseases.
\end{abstract}

\section{Graphical abstract}

\footnotetext{
*Corresponding Author: leafh@email.unc.edu.

Supporting Information

The Supporting Information is available free of charge on the ACS Publications website at DOI: 10.1021/acsami.6b05534

Physicochemical particle characteristics and in vitro studies as well as supplementary kinetics and distribution data (PDF)

Notes

The authors declare no competing financial interest.
} 

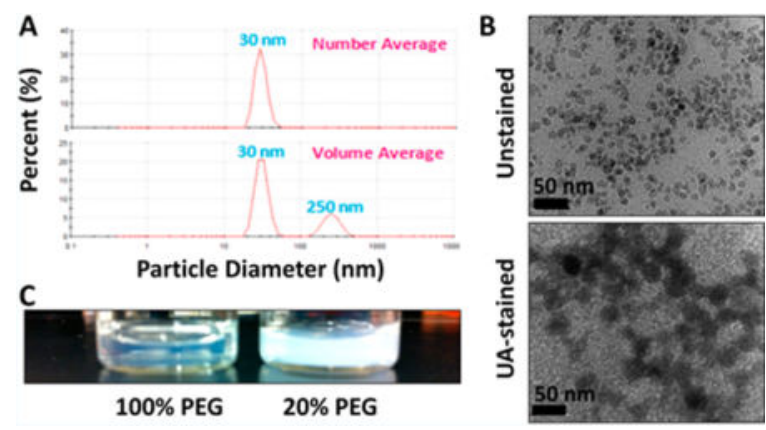

\section{Keywords}

liposome calcium phosphate; PEGylation; kinetics; distribution; tumor accumulation; lymphatic drainage

\section{INTRODUCTION}

Of the advances made upon liposomal platforms for drug and gene delivery over the last half-century, surface PEGylation remains one of the most foundational in conferring clinically meaningful effects. ${ }^{1,2}$ The ability to expand circulation longevity of liposomes through a decrease in opsonin-mediated immune cell recognition has allowed for a relevant exploitation of the enhanced permeation and retention (EPR) effect in cancer therapy. ${ }^{3-6}$ However, due to strong surfactant forces directed upon a PEGylated liposome surface, further compounded by the relatively weak hydrophobic exclusion effect which supports formation of a lipid bilayer around an aqueous core, PEGylation at greater than roughly 6-7 mol \% lipids serves to dissolve the lipid bilayer structure, producing lower-order micelles which possess more thermodynamically stable hydrophobic cores. ${ }^{7}$ Such limitations in density likely contribute to the rapid clearance of such nanoparticles compared to other advanced formulations by the immune system and may contribute to an acquired immune response upon subsequent injections, in particular toward the comparatively diverse chemical structure of the polyethylene glycol (PEG)-lipid conjugation site. ${ }^{8,9}$ Therefore, effective means by which to PEGylate liposomes to higher densities remain desirable in the development of advanced nanomedicines.

A series of recently developed liposomal formulations ${ }^{7,10,11}$ perform in such a manner by means of what is known as the "supported bilayer phenomenon". Specifically, should a liposome possess not an aqueous core, but a solid, stable, and molecularly interactive core material, the lipid bilayer will remain intact even under the forces conferred by increasing PEGylation density, allowing a progression toward the steric limits of PEGylation for each respective formulation. ${ }^{7}$ Considerable benefit has been wrought from the liposome calcium phosphate (LCP) formulation method in particular, including a selective and ApoEdependent tropism of LCP for the hepatocytes of the liver at such high PEGylation, ${ }^{12}$ as well as notable siRNA-mediated gene silencing efficacy in the systemic treatment of various cancers. ${ }^{13,14}$ However, despite high (20 mol \%) PEG density, previously published work often describes marginal circulation longevity for LCP, in particular as the macromolecular complexity of the encapsulated cargoes increases. ${ }^{12,15}$ Further, though cells possess reliable 
intrinsic means by which to overcome the high-calcium conditions elicited through LCPmediated endosome escape, ${ }^{16}$ cationic lipid-mediated toxicity remains a concern in many nanoparticle formulation strategies. ${ }^{3,17}$ LCP also has been shown to facilitate endosomal release even in the absence of cationic lipids through a number of coordinated mechanisms. ${ }^{12,18,19}$ Thus, an enhanced stealth property is expected to improve control over nanoparticle distribution in vivo, contributing to the therapeutic potential of such delivery systems overall.

Herein, we present for the first time a liposome coated on its outer leaflet exclusively by PEGylated phospholipids and representing a lipid packing density at the level of both inner and outer leaflets commensurate with a traditional lipid bilayer. These particles can be formulated with a variety of calcium phosphate $(\mathrm{CaP})$ core sizes $(10-15 \mathrm{~nm}, 30-40 \mathrm{~nm})$ and outer-leaflet polymer chain lengths (350-5000 Da) through a scalable resuspension process as well, avoiding the high-energy conditions required under such means for traditional liposome formulations. Such fully-PEGylated liposomes exhibit a chain length-dependent circulation longevity and an evasion of uptake in traditional organs of nanoparticle clearance (liver, kidneys, spleen), while maintaining passive tropism toward primary hepatocytes over Kupffer cells within the liver. ${ }^{12}$ In particular, due to their improved kinetic profile in vivo, these fully-PEGylated liposomes show both a strong accumulation within solid tumors upon intravenous injection and a more rapid and extensive local lymphatic access after subcutaneous administration. Given focused systemic distribution and capable cell internalization through traditional active targeting, these kinetically and distributively improved liposomes exhibit strong potential for the treatment of a variety of systemic and lymphatic diseases.

\section{MATERIALS AND METHODS}

\subsection{Materials}

1,2-Distearoyl-sn-glycero-3-phosphatidylethanolamine $-\mathrm{N} N$-[carbonyl-methoxy(polyethylene glycol)-2000] (DSPE-PEG2000) was purchased from NOF corporation (Tokyo, Japan). 1,2Dioleoyl-sn-glycero-3-phosphate (DOPA), 1,2-dioleoyl-sn-glycero-3-phosphocholine (DOPC), and 1,2-dioleoyl-3-trimethylammonium-propane (DOTAP) were purchased from Avanti Polar Lipids, Inc. (Alabaster, AL). DSPE-PEG 2000 -anisamide (AA), DSPE$\mathrm{PEG}_{2000}$-galactose (GAL), and DSPE-PEG 2000 -mannose (MAN) were synthesized following previously described protocols ${ }^{15,20,21}$ and analyzed via NMR using a Varian Inova $400 \mathrm{MHz}$ NMR spectrometer (Palo Alto, CA). All other lipids, including nitrobenzoxadiazolyl (NBD)- and fluorescein isothiocyanate (FITC)-modified DOPA, DOPC, and DSPE-PEG (referenced in the text as NBD-OPA, NBD-OPC, and DSPE-PEGFITC, respectively), were purchased from Avanti Polar Lipids, Inc. (Alabaster, AL). Ultracentrifuge tubes were purchased from Beckman Coulter (Indianapolis, IN). ${ }^{177} \mathrm{Lu}$ was purchased from PerkinElmer (Waltham, MA). All other reagents, including fluorescently labeled oligonucleotides, were purchased from Sigma-Aldrich (St. Louis, MO) and used without further purification. Six- to eight-week-old BALB/c female mice were purchased from Charles River Laboratories (Wilmington, MA). All work performed on animals was in 
accordance with and approved by the University of North Carolina Institutional Animal Care and Use Committee.

\subsection{Preparation and Characterization of Fully-PEGylated LCP Nanoparticles}

LCPs were prepared using a modified protocol. ${ }^{12,22}$ Briefly, two, 5 mL 3:7 Igepal co-520/ cyclohexane w/o microemulsions were prepared: one with $150 \mu \mathrm{L}$ of dibasic ammonium phosphate $(50 \mathrm{mM})$ and the other with $150 \mu \mathrm{L}$ of calcium chloride $(2.5 \mathrm{M})$ (with or without soluble therapeutic). Emulsions were stirred for $10 \mathrm{~min}$, mixed together, and stirred for an additional $10 \mathrm{~min}$, followed by addition of $62.5 \mu \mathrm{L}$ of DOPA (35 mM). After an additional 30 min of stirring, the mixture was washed repeatedly with absolute ethanol under centrifugation $(10000 \mathrm{~g})$ to yield a precipitate purified from the excess surfactants. The precipitate was then resuspended in chloroform and centrifuged once more $(10000 \mathrm{~g})$ to purify the DOPA-coated calcium phosphate $(\mathrm{CaP})$ cores (supernatant) from more poorly formed nanoprecipitates (pellet). Encapsulation was characterized radiometrically using a gamma counter (the Nucleus, Oak Ridge, TN) or through a fluorescence method.

$\mathrm{CaP}$ cores were then mixed with a slight (2.5-fold) excess of outer-leaflet lipids in chloroform, dried into a thin, transparent film under nitrogen, and resuspended at $65{ }^{\circ} \mathrm{C}$ under aqueous conditions (beginning with absolute ethanol and titrating to $<20 \%$ with additional DI water). Molar ratios of outer-leaflet lipids for the traditional LCP formulation follow as 2:2:1 DOTAP/cholesterol/DSPE-PEG. Zeta potential and particle size were measured using a Malvern ZetaSizer Nano Series (Westborough, MA). TEM images of both DOPA-coated CaP cores and fully-PEGylated LCP liposomes were acquired using a JEOL 100CX II TEM (JEOL, Japan), the latter of which was stained with uranyl acetate (UA).

For surface composition analysis, LCP purification was performed via sucrose density gradient centrifugation. Briefly, in a centrifuge tube, layers of varying sucrose concentration $(40 \%, 20 \%, 10 \%$, and $0 \%)$ were prepared, with nanoparticle solution comprising the top layer. Nanoparticles were labeled at the level of the core $\left({ }^{177} \mathrm{Lu}\right)$ and either the inner leaflet (0.3\% NBD-OPA) or the outer leaflet (1\% NBD-OPC or 1\% DSPE-PEG-FITC). Samples were centrifuged using a swinging bucket rotor $\left(60000 \mathrm{rpm}, 4 \mathrm{~h}, 4{ }^{\circ} \mathrm{C}\right)$, and visible fluorescent signal under UV illumination, which penetrated into the lower sucrose layers, was recovered. Such fractions were analyzed fluorometrically to determine the percentage of input fluorescence recovered (through either the CaP core preparation for NBD-OPA or the density gradient centrifugation for the NBD-OPC or DSPE-PEG-FITC) and normalized radiometrically to core content.

\subsection{Pharmacokinetics and Biodistribution of Fully-PEGylated LCP Nanoparticles}

$\mathrm{BALB} / \mathrm{c}$ mice were injected intravenously (via tail vein) or subcutaneously (local to the right inguinal lymph node) with nanoparticle suspensions (supplemented with 5\% glucose to balance osmolarity). For kinetics studies, blood samples were extracted serially via tail clip, with the final time point obtained via cardiac puncture. For studies in tumor-bearing mice, orthotopic 4T1 tumors were produced in BALB/c mice through subcutaneous inoculation of $2 \times 10^{5}$ cells at the mammary fat pad and grown under monitoring over the following $8-10$ days. Organ harvest was conducted at $6,24,48$, or $72 \mathrm{~h}$ postinjection, depending on the 
kinetic longevity of the nanoparticles in question; all solid organs were rinsed with PBS and dried with lab tissues before weighing. Blood samples and whole organ extracts were characterized radiometrically using a gamma counter.

\subsection{Hepatic Uptake of Fluorescence-Labeled Oligonucleotides Delivered by Fully- PEGylated LCP}

Fully-PEGylated LCP nanoparticles encapsulating Texas Red-labeled oligonucleotides were injected intravenously in 5\% glucose to balance osmolarity. After $12 \mathrm{~h}$, livers were harvested and fixed in formalin overnight, hydrated in PBS containing $30 \%$ sucrose, and frozen in OCT (Tissue-Tek, Dublin, OH). Ten micron tissue sections were generated and mounted onto Superfrost Plus slides (Fisher Scientific, Houston, TX). Tissue sections were rinsed with PBS and mounted in DAPI-containing medium; actin staining was facilitated prior to addition of mounting medium via FITC-phalloidin staining and subsequent washing with PBS. Images were acquired with a Nikon Eclipse Ti fluorescence microscope.

\subsection{Statistical Analysis}

Data are presented as mean $\pm \mathrm{SD}$. Statistical significance was defined by $P<0.05$ through a two-tailed $t$ test when comparing two groups and the Holm-Sidak test for multiple comparisons.

\section{RESULTS AND DISCUSSION}

\subsection{LCP Nanoparticles Coated Exclusively by PEGylated Phospholipids: Preparation, Characterization, and Surface Density Analysis}

Lipid-coated nanoparticle $(\mathrm{CaP})$ cores have proven to be an effective formulation mechanism for drug encapsulation and delivery, in particular for a variety of nucleic acid and phosphorylated peptide and prodrug therapies which are limited in formulation within traditional liposomes. ${ }^{15,21,23}$ Unique to such CaP cores is the dense coating of DOPA on the calcium phosphate nanoparticle surface, which is underscored by the maintenance of such high-density particles (theoretical specific activity of $\sim 1.6$ ) in suspension in chloroform under high separation forces (centrifugation at $10000 \mathrm{~g}$ ). Previous methods for generating an outer lipid layer on such $\mathrm{CaP}$ cores employed a mixed lipid system comprising traditional bilayer-forming lipids such as DOPC and DOTAP, helper lipids such as cholesterol, and the antifouling polymer-lipid conjugate DSPE-PEG 2000 ; within such a thermodynamic system, PEG density on the nanoparticle surface has been shown to plateau at roughly $20 \mathrm{~mol} \%$ of total outer lipid. ${ }^{12}$ However, CaP core coating exclusively with PEGylated phospholipids has yet to be explored. Herein, we present such liposomes for the first time, coating nanoparticle cores ranging from 10 to $15 \mathrm{~nm}$ in diameter (Figure 1B) exclusively with DSPE-PEG 2000 to produce a well-dispersed population of around $30 \mathrm{~nm}$ (Figures 1A,B and S1B). Such particles represent only minor degrees of aggregation, as observed instead through a volumeaveraged distribution (Figure 1A) as well as a visually transparent final product compared to traditional $20 \mathrm{~mol} \%$-PEGylated LCP (Figure 1C). Most notable for this fully-PEGylated formulation is the ease with which one can resuspend such nanoparticles from a transparent thin film containing DSPE-PEG 2000 through simple addition of fresh, absolute ethanol followed by rapid dilution with DI water, avoiding the high-energy and comparatively scale- 
limited disruptive methods (i.e., sonication) of deaggregation typically employed for the traditional 20 mol \%-PEGylated LCP formulation at comparable outer leaflet excesses. Further, such exclusive phospho-lipopolymer coatings can be formed with a variety of PEGylation chain lengths (Figure S1A) and CaP core sizes (Figure S1B) through the inclusion of cholesterol in the formulation.

Traditional bilayer-forming lipids such as DOPA (under deionized conditions) or DOPC, when assembled into a lipid bilayer structure, possess headgroup cross-sectional areas (CSA) on the sub-nm ${ }^{2}$ level. ${ }^{24}$ Further, a headgroup CSA in great excess of that of the acyl chains will drive micellar formulation in aqueous suspension, as in the case of DSPE-PEG alone, preventing the full PEGylation of a traditional liposome possessing an aqueous core. Given the stability of our $\mathrm{CaP}$ cores in suspension under high separation forces and our ability to formulate $\mathrm{CaP}$ cores exclusively on the outer leaflet with DSPE-PEG, we desired to characterize the lipid packing density on the surface of the calcium phosphate precipitates, in terms of proposed inner leaflet and outer leaflet. Given the obvious thermodynamic discrepancies between traditional liposomes and LCP, as well as the significant reduction in size of our formulations with regard to the capabilities of traditional liposomes, our goals herein were limited to ascertaining whether DOPA within the inner leaflet and/or DSPE-PEG 2000 on the outer leaflet organizes on the surface of the calcium phosphate cores in a density commensurate with traditional bilayer-forming lipids. To characterize these nanoparticles at each level of the formulation, we expanded upon an established protocol of density-gradient centrifugation, ${ }^{12}$ utilizing the following components for quantitation: ${ }^{177} \mathrm{Lu}$, a radionuclide whose solubility product equilibrium favors encapsulation within the $\mathrm{CaP}$ core; NBD-OPA, a fluorescent lipid used in small proportions to ascertain the DOPA content on the surface of the CaP core; NBD-OPC, a fluorescent analogue of DOPC which functions similarly for outer leaflet quantitation; and DSPE$\mathrm{PEG}_{2000}$-FITC. Thus, at each level of the particle (core, inner leaflet, outer leaflet, PEG corona), we possess a means by which to characterize the particles after purification under sucrose density gradient, wherein the nanoparticles accumulate as a narrow band (Figure S1B) and can be harvested and analyzed both radiometrically and fluorometrically. Table 1 summarizes these measurements based on the fractional densities of both calcium phosphate and oleic acid within the $12 \mathrm{~nm} \mathrm{CaP}$ cores (Figure 1B). Therein, we observe PA, PC, and PE headgroup CSA on the subnm ${ }^{2}$ level, which suggests a density of lipid packing commensurate with traditional bilayer formation for both traditional LCP as well as its fullyPEGylated counterpart, despite working with a nanoparticle for which the aqueous core analogue (a liposome approximately $20 \mathrm{~nm}$ in diameter) would be entirely unstable. These data represent the first determination of lipid packing density for the LCP system as well.

Such results may at first glance seem surprising for a bilayer system which exclusively incorporates DSPE-PEG on the outer leaflet, as they suggest a relatively high density of PEG (1.3 PEG chains per $\mathrm{nm}^{2}$ ) on a spontaneously organized surface. However, the high aspect ratio of such nanoparticle cores $(\sim 10 \mathrm{~nm})$ must be emphasized in this formulation in comparison to traditional liposomal formulations such as Doxil $(\sim 100 \mathrm{~nm})$. As the PEG corona occupies a majority of the total volume of the fully-PEGylated LCP, one can clearly envision the multiple-fold increases in free volume as one expands outward radially, along with the underlying entropic benefits. Thus, at the distal ends of the PEG corona on these 
PEGylated LCP nanoparticles (a 5-10 nm radial expansion as observed by DLS and a relatively conservative benchmark for a brush-conforming PEGylation density), one can easily consider a far less densely packed polymer system, particularly where entropic benefit is most easily accessible for the polymer chains. Moreover, this expansion of free volume is most evident when considering the larger $\mathrm{CaP}$ cores (Figure S1B), which cannot be resuspended effectively without the presence of a lesser proportion of cholesterol (2:1 PEG/ chol). Larger cores of 30-40 $\mathrm{nm} \mathrm{CaP}$ diameter possess far less of an increase in aspect ratio as one expands outward radially; thus, cholesterol, which possesses non-negligible surface area occupancy in its own right within the bilayer, serves to expand the initial free volume occupied by the PEG chains and support entropic gains at the level of the lipid bilayer rather than at the PEG corona. Further studies to thoroughly characterize the lipid character on such nanoparticles remain necessary; however, a bilayer organization of lipids represents the most reasonable interpretation of the LCP nanoparticle surface.

\subsection{Kinetics and Biodistribution of Fully-PEGylated LCP after Intravenous Injection}

Our motivation to increase further the density of PEG on the LCP nanoparticle surface initiated in part from previous work with traditional LCP, which showed a surprisingly rapid distribution clearance, only exhibiting extended circulation longevity after well over half of the injected dose had been eliminated from the circulation. ${ }^{12,15}$ Further, the LCP nanoparticle system, in particular due to the novelty in establishing a supported lipid bilayer on the surface of a solid CaP nanoparticle core, has been shown to open up a variety of therapeutics (nucleic acids, ${ }^{10,13,15}$ phosphorylated peptides, ${ }^{21}$ and small-molecule prodrugs ${ }^{13}$ ) to unilamellar liposomal delivery and efficacy in vivo which are exceedingly difficult to load passively within the aqueous core environment of a traditional liposome (e.g., Doxil). Given our particles' increased PEGylation density on the outer leaflet, as well as the improved quality of the formulation (particularly without extensive excesses of outer leaflet lipids or extreme formulation conditions such as sonication), we hypothesized that we would be able to reduce such rapid distribution clearance and increase the overall systemic exposure to our fully-PEGylated formulation. Further, we sought to broaden our efforts in exploring the impact of PEG chain length on circulation longevity at such unprecedented liposomal PEGylation density. After injection via tail vein, BALB/c mice were sampled serially for blood at various time points and sacrificed for whole-organ biodistribution analysis after sufficient clearance from the circulation. Figure 2A highlights such chain length dependencies on circulation longevity, showing therein a proportional relationship. While LCP coated exclusively with $\mathrm{PEG}_{350}$ phospholipids was rapidly cleared from the circulation, upon extension of the chain to $\mathrm{PEG}_{1000}$, a two-phase distribution profile emerged. Further extension to $\mathrm{PEG}_{2000}$ decreased both the distribution and elimination clearance rates as well, with $\mathrm{PEG}_{5000}$ allowing for only a negligible increase in overall exposure. Further, such increases in kinetic longevity (a mean residence time of $13 \mathrm{~h}$ for the $\mathrm{PEG}_{2000}$ formulation) correlate as expected to the extent of protein adsorption to the nanoparticles (Figure S2A), which is readily anticipated given the marked differences between such formulations in their ability to effectively screen liposome surface charge from the surrounding environment (Table S1). Further, such fully-PEGylated LCP nanoparticles encapsulating both model drug (dsDNA oligos) and ${ }^{177} \mathrm{Lu}$ almost fully abrogate the distribution clearance which 20 mol \% PEG $_{2000}$-modified LCP possesses, particularly under 
low-energy resuspension conditions, increasing the overall systemic exposure to the nanoparticles (Figure S2B). In contrast to previously published data as well, ${ }^{15}$ targeting to the asialoglycoprotein (ASGPR) receptor on hepatocytes through galactose ligands (conjugated to the distal chain end of PEG) contributed to an increased distribution clearance without entirely abrogating nanoparticle long-circulating properties. Further, $\mathrm{CaP}$ core size does not meaningfully impact nanoparticle circulation or biodistribution properties (Figure $\mathrm{S} 2 \mathrm{C})$.

These dependencies on chain length and targeting express themselves further through the terminal biodistribution profiles of such fully-PEGylated particles (Figure 2B). As one increases PEG chain length from 350 to 1000 to $2000 \mathrm{Da}$, dramatic changes in their overall distribution to major organs of nanoparticle clearance are apparent. Given their instability in physiological salt and serum conditions due to their failure at effective liposome surface charge screening, $\mathrm{PEG}_{350}$-modified LCP exhibits an extensive distribution to the liver, where over half of the injected radionuclide accumulates. While PEG $_{1000}$-modified LCP shows a prolonged clearance and an enhanced evasion of uptake within the liver, splenic accumulation predominates at a surprising 304\% ID per gram tissue; however, such phenomena of dramatic splenic nanoparticle accumulation is not entirely unexpected as one progresses into the long-circulation domain. ${ }^{25}$ It is sufficient, however, at the level of $\mathrm{PEG}_{2000}$ modification for LCP to effectively evade uptake in all major clearing organs, instead accumulating in bone at anywhere from $10 \%$ to $25 \%$ ID per gram in our sampling due to the natural tropism for free Lu to accumulate therein (such high accumulation, extended across the entire skeletal system, corresponds to roughly half of the injected ${ }^{177} \mathrm{Lu}$ dose). To confirm this evaluation mechanistically, galactose- $\mathrm{PEG}_{2000}$-modified LCP resolves much of the loss of ${ }^{177} \mathrm{Lu}$ in this manner, refocusing the nanoparticles more specifically to the liver $(\sim 50 \% \mathrm{ID} / \mathrm{g})$, on par with the traditional LCP formulation ${ }^{15}$ through active targeting (in contrast to the passive scavenging of the poorly shielded $\mathrm{PEG}_{350}$ formulation by the liver). In essence, this natural bone tropism for our radionuclide tracer serves a dual benefit of tracking a truly particle-specific distribution while also providing means through which to quantitatively characterize such particles' stealth properties, given the ability for LCP modified with increasing PEG chain length to avoid uptake within traditional organs of nanoparticle clearance. The $\mathrm{PEG}_{5000}$ formulation functioned similarly to the $\mathrm{PEG}_{2000}$ formulation, with minor accumulation differences potentially owing a causal relationship to the well-considered PEG shedding phenomenon, whereby over extended in vivo exposure lipids with an increased ratio between PEG and acyl chain length will shed such chains at an increased rate, thus opening up such particles to opsonization and immune scavenging despite long-circulating benefits. ${ }^{3,26}$ Further, our supported bilayer platform provides a mechanism by which to fully PEGylate the outer leaflet utilizing macromolecular materials which would self-organize into micelles under any alternative conditions of spontaneous self-assembly. Thus, our LCP platform can be confirmed to support a functional outer leaflet independent of phyisco-chemical considerations which drive traditional liposome and polymersome formation. 


\subsection{Hepatic Distribution of Fully-PEGylated LCP after Intravenous Injection}

Given such improved circulation properties and enhanced stealth supported by such fullyPEGylated liposomes, we sought to explore the means through which such a particle system may support favorable distribution to a variety of therapeutic targets in vivo; namely, both primary hepatocytes and solid tumors via systemic delivery and local as well as systemic lymphatics upon subcutaneous administration. In the case of the former, previous studies utilizing LCP PEGylated to low densities ( $0-20 \mathrm{~mol} \%)$ presented a surprising phenomenon which was found to be independent of active targeting: the dramatic alteration in the passive distribution of such nanoparticles locally within the liver, showing a shift in tropism with increased PEGylation density beyond $10 \mathrm{~mol} \%$ from Kupffer cells, the resident macrophages of the liver, to uptake within the primary hepatocytes. ${ }^{12} \mathrm{We}$ thus sought to explore whether a fully-PEGylated LCP nanoparticle, delivering a Texas Red-labeled oligonucleotide, could extend this phenomenon absent any traditional bilayer-forming lipids or active targeting strategies. Mice were sacrificed at $12 \mathrm{~h}$ postinjection (after extensive clearance of the nanoparticles from the circulation), and livers were harvested, fixed, sectioned, and stained with both DAPI (blue) and FITC-phalloidin (green) for visualization of nucleus and cytoskeleton, respectively. Indeed, through fluorescence imaging of these frozen liver sections (Figure 3), we observe a notable delivery of Texas Red-labeled dsDNA oligo through the fully-PEGylated formulation; further, such distribution is represented as a diffuse red fluorescence across the hepatocellular substructures, indicating an effective escape of such oligonucleotides from endosomes after internalization. Thus, LCP nanoparticles coated exclusively on their outer leaflet by DSPE-PEG 2000 can also facilitate comparably hepatocyte-specific delivery at the intraorgan level, extending the phenomenon observed through traditional LCP.

\subsection{Accumulation of Fully-PEGylated LCP upon Local and Systemic Administration within Local and Systemic Lymphatics and Solid Tumors}

Next, we sought to explore the potential for such particles in delivery both locally and systemically toward more complex targets. With complete PEGylation of the outer leaflet in our novel formulation, we anticipated an improved lymphatic drainage and accumulation over time upon subcutaneous administration, ${ }^{23}$ as a logical extension of phenomena observed in traditional liposomal PEGylation ${ }^{27}$ as well as alternative particle systems such as dendrimers (which in particular exhibit a decreased systemic and increased lymphatic distribution with both an increase in particle size and PEGylation). ${ }^{28}$ Therefore, BALB/c mice were injected s.c. with either 100 or 20 mol \% PEGylated particles in a 5\% glucose solution, each targeted with $20 \%$ of the total DSPE- $\mathrm{PEG}_{2000}$ functionalized as mannosemodified DSPE-PEG 2000 , and major organs were harvested after 6 and $24 \mathrm{~h}$ to characterize particle accumulation therein. Though systemic kinetic absorption was not detectable for such formulations, accumulation for the fully-PEGylated (PCP) (Figure 4) within the draining lymph node was increased over 6-fold (970\% vs 150\% ID per gram tissue). However, the fully-PEGylated yet untargeted formulation exhibited significant accumulation within systemic lymphatics (specifically, within the contralateral lymph node to the injection site), implying meaningful absorption characteristics absent local internalization. Therefore, to compare with the glucose-balanced particles, we instead prepared an osmotically balanced preparation in $10 \%$ sucrose, intended for the inhibition of clathrin-mediated 
endocytosis local to the injection site. Indeed, we can observe dramatic absorption of both formulations within the systemic circulation in this capacity, a recapitulation of the lymphatic accumulation contralateral to the injection site, and a consistent maintenance of the differential accumulation within the draining inguinal lymph node between 20 and 100 mol \% PEGylated formulations (Figure S3), emphasizing the impact of targeting on particle distribution in vivo. Further, such fully-PEGylated particles remain amenable to internalization into an immortalized dendritic cell line (JAWSII) to improved degrees to that of 20 mol \% PEGylated LCP (Figure S5). Therefore, such exclusively PEGylated nanoparticles represent an improved delivery system for accumulation in local lymphatics upon subcutaneous administration.

Similarly, such a prolonged distribution phase and stealth property for the fully-PEGylated formulation would be expected to benefit accumulation within solid tumors via systemic administration, in part through the well-characterized enhanced permeability and retention (EPR) effect. ${ }^{6} \mathrm{BALB} / \mathrm{c}$ mice were inoculated with $4 \mathrm{~T} 1$ cells orthotopically at the mammary fat pad, where after $8-10$ days tumors varying in size around $100 \mathrm{~mm}^{3}$ were formed. Mice were then injected i.v. with PCP or LCP particles, either unmodified or incorporating $20 \%$

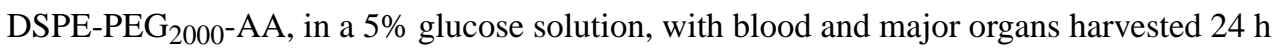
postinjection. Overall PCP nanoparticle distribution within the host as well as to solid tumor remained unaffected by targeting and produced a particle-specific distribution profile representing accumulation within tumors $(\sim 10-12 \% \mathrm{ID} / \mathrm{g})$ on par with hepatic and splenic accumulation ( 15-30\% ID/g) (Figure 5). While LCP nanoparticles displayed only a modest difference in their accumulation within tumors $(\sim 5 \% \mathrm{ID} / \mathrm{g})$, the dramatic accumulation of such nanoparticles within the liver and spleen ( $\sim 60 \%$ and $150 \% \mathrm{ID} / \mathrm{g}$, respectively) underscores the value which PCP provides in its stealth property, as can be observed in the accumulation ratios between tumor and either liver or spleen. Further, PCP particles again remain amenable to internalization to improved degrees to that of traditional LCP (Figure S5). Therefore, such fully-PEGylated nanoparticles also represent an effective delivery system for accumulation within solid tumors upon intravenous administration. In each of these cases of local and systemic delivery, we are currently evaluating the therapeutic efficacy of these fully-PEGylated liposomes and plan to include such findings in a later publication.

\section{CONCLUSIONS}

In this work, we present for the first time a liposome based on the lipid-coated calcium phosphate $(\mathrm{CaP})$ core platform of diverse sizes $(10-15 \mathrm{~nm}, 30-40 \mathrm{~nm})$ that is coated on the outer leaflet exclusively by PEGylated phospholipids of varying polymer chain length (350$5000 \mathrm{Da}$ ). Such LCP particles exhibit improved circulation longevity and immune-evasive properties in a chain length-dependent manner, while facilitating both strong accumulation within solid tumors upon intravenous injection and a more rapid and extensive lymphatic drainage upon subcutaneous administration. Further, these fully-PEGylated LCP particles remain amenable to active targeting strategies which elicit improved degrees of focused distribution and nanoparticle uptake, represent an outer leaflet surface density commensurate with the formation of a lipid bilayer, and avoid harsh physical resuspension processes which are necessary for preparation of traditional LCP nanoparticles. We anticipate that such 
improved delivery properties can translate into improved therapeutic safety and efficacy for a variety of systemic and lymphatic diseases.

\section{Supplementary Material}

Refer to Web version on PubMed Central for supplementary material.

\section{Acknowledgments}

The authors would like to thank CHANL at UNC for access to TEM instrumentation. The authors would also like to thank Drs. Yang Liu, Yu-Cheng Tseng, and Yunxia Hu, whose previous work on LCP inspired these efforts herein. This work was supported by NIH Grants CA149387, CA198999, and DK100664, with fellowship funding for M.T.H. provided by the Royster Society of Fellows at UNC and the American Foundation for Pharmaceutical Education (AFPE).

\section{References}

1. Klibanov AL, Maruyama K, Torchilin VP, Huang L. Amphipathic Polyethyleneglycols Effectively Prolong the Circulation Time of Liposomes. FEBS Lett. 1990; 268:235-237. [PubMed: 2384160]

2. James ND, Coker RJ, Tomlinson D, Harris JRW, Gompels M, Pinching AJ, Stewart JSW. Liposomal Doxorubicin (Doxil): An Effective New Treatment for Kaposi's Sarcoma in AIDS. Clin Oncology. 1994; 6:294-296.

3. Huang L, Liu Y. In Vivo Delivery of RNAi with Lipid-Based Nanoparticles. Annu Rev Biomed Eng. 2011; 13:507-530. [PubMed: 21639780]

4. Torchilin V. Tumor Delivery of Macromolecular Drugs Based on the EPR Effect. Adv Drug Delivery Rev. 2011; 63:131-135.

5. Maruyama K. Intracellular Targeting Delivery of Liposomal Drugs to Solid Tumors Based on EPR Effects. Adv Drug Delivery Rev. 2011; 63:161-169.

6. Maeda H, Wu J, Sawa T, Matsumura Y, Hori K. Tumor Vascular Permeability and the EPR Effect in Macromolecular Therapeutics: A Review. J Controlled Release. 2000; 65:271-284.

7. Li S-D, Huang L. Nanoparticles Evading the Reticuloendothelial System: Role of the Supported Bilayer. Biochim Biophys Acta, Biomembr. 2009; 1788:2259-2266.

8. Ishida T, Kiwada H. Accelerated Blood Clearance (ABC) Phenomenon upon Repeated Injection of PEGylated Liposomes. Int J Pharm. 2008; 354:56-62. [PubMed: 18083313]

9. Shiraishi K, Hamano M, Ma H, Kawano K, Maitani Y, Aoshi T, Ishii KJ, Yokoyama M. Hydrophobic Blocks of PEG-conjugates Play a Significant Role in the Accelerated Blood Clearance (ABC) Phenomenon. J Controlled Release. 2013; 165:183-90.

10. Li J, Yang Y, Huang L. Calcium Phosphate Nanoparticles with an Asymmetric Lipid Bilayer Coating for siRNA Delivery to the Tumor. J Controlled Release. 2012; 158:108-114.

11. Guo S, Wang Y, Miao L, Xu Z, Lin CM, Zhang Y, Huang L. Lipid-Coated Cisplatin Nanoparticles Induce Neighboring Effect and Exhibit Enhanced Anticancer Efficacy. ACS Nano. 2013; 7:98969904. [PubMed: 24083505]

12. Liu Y, Hu Y, Huang L. Influence of Polyethylene Glycol Density and Surface Lipid on Pharmacokinetics and Biodistribution of Lipid-Calcium-Phosphate Nanoparticles. Biomaterials. 2014; 35:3027-3034. [PubMed: 24388798]

13. Zhang Y, Schwerbrock NMJ, Rogers AB, Kim WY, Huang L. Codelivery of VEGF siRNA and Gemcitabine Monophosphate in a Single Nanoparticle Formulation for Effective Treatment of NSCLC. Mol Ther. 2013; 21:1559-1569. [PubMed: 23774791]

14. Yang Y, Li J, Liu F, Huang L. Systemic Delivery of siRNA via LCP Nanoparticle Efficiently Inhibits Lung Metastasis. Mol Ther. 2012; 20:609-615. [PubMed: 22186791]

15. Hu Y, Haynes MT, Wang Y, Liu F, Huang L. A Highly Efficient Synthetic Vector: Nonhydrodynamic Delivery of DNA to Hepatocyte Nuclei in Vivo. ACS Nano. 2013; 7:53765384. [PubMed: 23647441] 
16. Tseng Y-C, Yang A, Huang L. How Does the Cell Overcome LCP Nanoparticle-Induced Calcium Toxicity? Mol Pharmaceutics. 2013; 10:4391-4395.

17. Zhang SB, Zhao B, Jiang HM, Wang B, Ma BC. Cationic Lipids and Polymers Mediated Vectors for Delivery of siRNA. J Controlled Release. 2007; 123:1-10.

18. Fujita T, Furuhata M, Hattori Y, Kawakami H, Toma K, Maitani Y. Calcium Enhanced Delivery of Tetraarginine-PEG-Lipid-Coated DNA/Protamine Complexes. Int J Pharm. 2009; 368:186-192. [PubMed: 18996454]

19. Sonawane ND, Szoka FC Jr, Verkman AS. Chloride Accumulation and Swelling in Endosomes Enhances DNA Transfer by Polyamine-DNA Polyplexes. J Biol Chem. 2003; 278:44826-31. [PubMed: 12944394]

20. Banerjee R, Tyagi P, Li S, Huang L. Anisamide-targeted Stealth Liposomes: A Potent Carrier for Targeting Doxorubicin to Human Prostate Cancer Cells. Int J Cancer. 2004; 112:693-700. [PubMed: 15382053]

21. Xu Z, Ramishetti S, Tseng Y-C, Guo S, Wang Y, Huang L. Multifunctional NanoparticlesCodelivering Trp2 Peptide and CpG Adjuvant Induce Potent Cytotoxic T-lymphocyte Response against Melanoma and its Lung Metastasis. J Controlled Release. 2013; 172:259-265.

22. Liu Y, Tseng Y-c, Huang L. Biodistribution Studies of Nanoparticles Using Fluorescence Imaging: A Qualitative or Quantitative Method? Pharm Res. 2012; 29:3273-3277. [PubMed: 22806405]

23. Tseng Y-C, Xu Z, Guley K, Yuan H, Huang L. Lipid-Calcium Phosphate Nanoparticles for Delivery to the Lymphatic System and SPECT/CT Imaging of Lymph Node Metastases. Biomaterials. 2014; 35:4688-4698. [PubMed: 24613050]

24. Dickey A, Faller R. Examining the Contributions of Lipid Shape and Headgroup Charge on Bilayer Behavior. Biophys J. 2008; 95:2636-2646. [PubMed: 18515396]

25. Owens DE Iii, Peppas NA. Opsonization, Biodistribution, and Pharmacokinetics of Polymeric Nanoparticles. Int J Pharm. 2006; 307:93-102. [PubMed: 16303268]

26. Li S-D, Huang L. Stealth Nanoparticles: High Density but Sheddable PEG is a Key for Tumor Targeting. J Controlled Release. 2010; 145:178-181.

27. Cai S, Yang Q, Bagby T, Forrest ML. Lymphatic Drug Delivery Using Engineered Liposomes and Solid Lipid Nanoparticles. Adv Drug Delivery Rev. 2011; 63:901-908.

28. Kaminskas LM, Kota J, McLeod VM, Kelly BD, Karellas P, Porter CJH. PEGylation of Polylysine Dendrimers Improves Absorption and Lymphatic Targeting Following SC Administration in Rats. J Controlled Release. 2009; 140:108-116. 

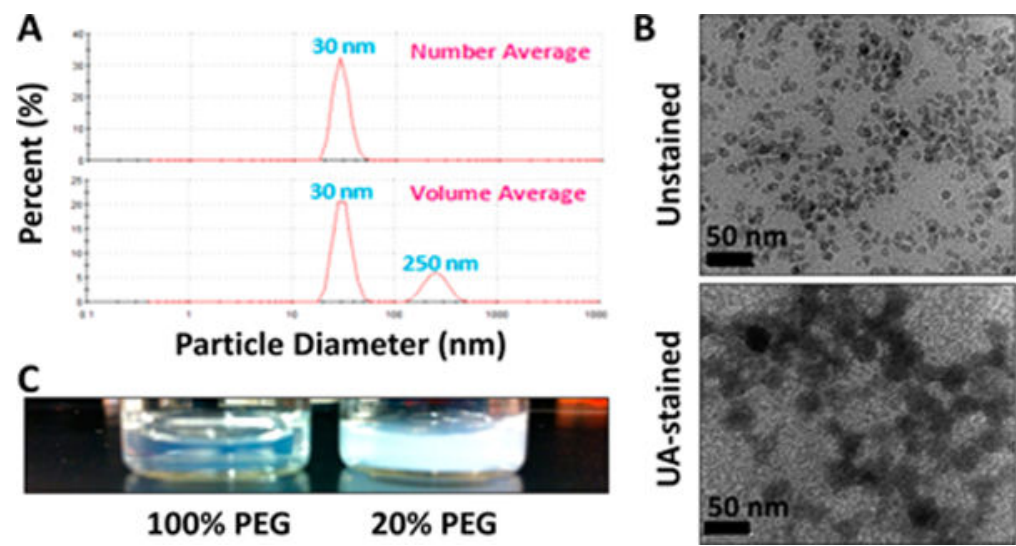

Figure 1.

LCP coated exclusively by PEGylated phospholipids. LCP cores were rehydrated from a clear, transparent thin film under high ethanol conditions, with optimal resuspension occurring with fresh absolute ethanol at $65^{\circ} \mathrm{C}$, followed by rapid dilution with deionized water. TEM images (B) and DLS measurements (A) convey a well-dispersed and highly transparent $\mathrm{CaP}$ core population in comparison to traditional LCP (20 mol \% PEGylation, (C)). UA = uranyl acetate. Scale bar $=50 \mathrm{~nm}$. 

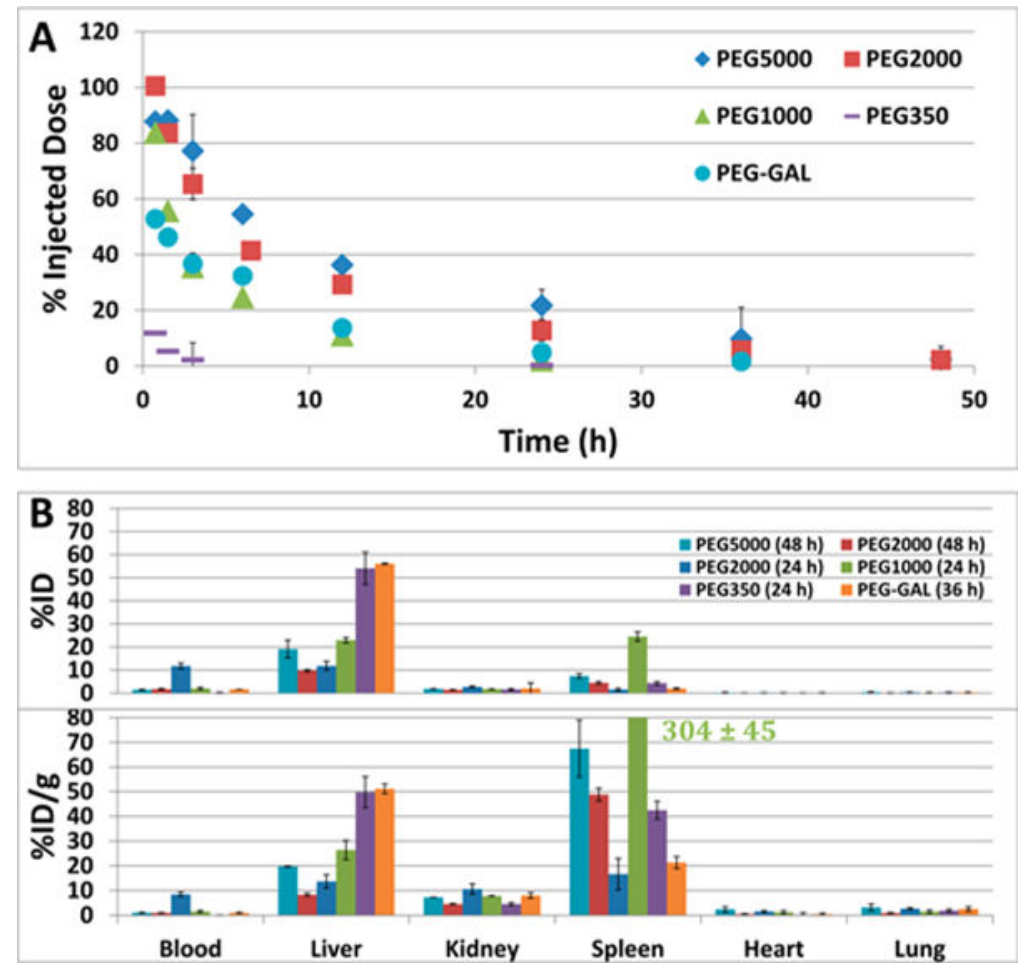

Figure 2.

Kinetics and biodistribution of fully-PEGylated LCP liposomes exhibits chain lengthdependent stealth properties. CaP precipitates encapsulating trace amounts of 177Lu were modified exclusively with PEGylated phospholipids (DSPE) of varying chain length and targeting capabilities and administered via tail vein to BALB/c mice $(n=4)$, with kinetics (A) monitored radiometrically through serial sampling and biodistribution (B) similarly after organ harvest. PEG-GAL: ASGPR-targeted PEG2000. 


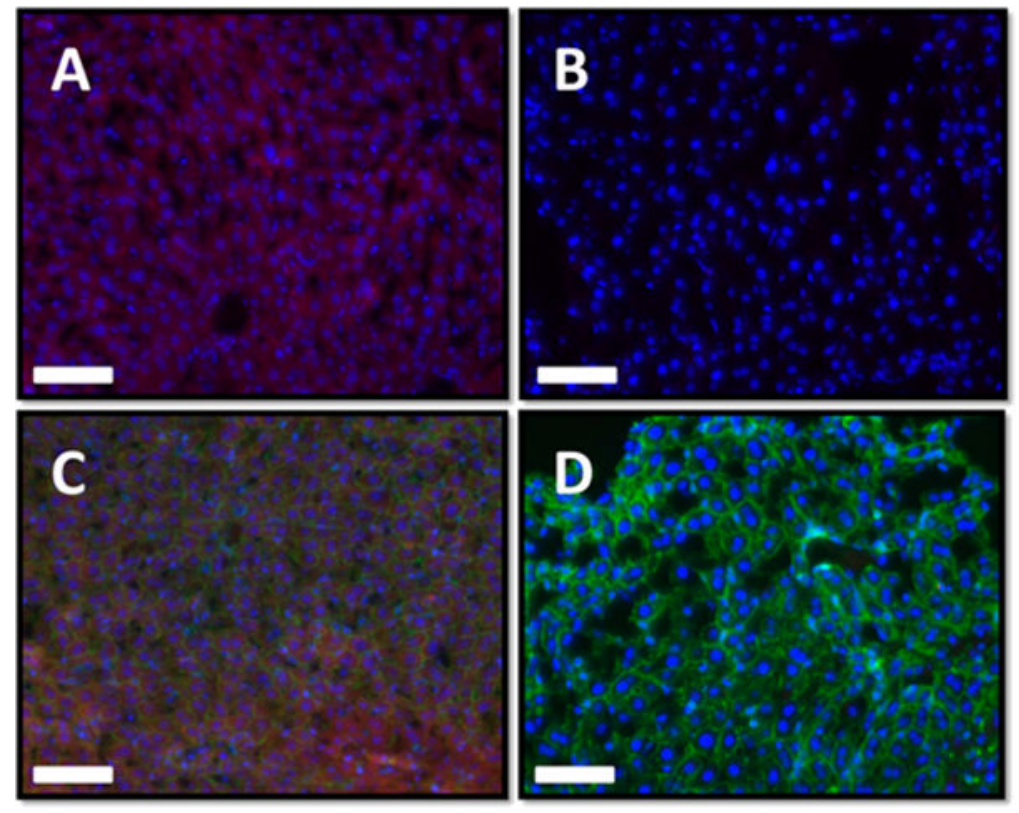

Figure 3.

Primary hepatocyte targeting through a fully-PEGylated LCP. DSPE-PEG $2 \mathrm{k}$-modified LCP encapsulating oligonucleotides labeled with Texas Red were administered via tail vein. Twelve hours later, BALB/c mouse livers were harvested and frozen sections were prepared for imaging. Green = FITC-phalloidin stain. Blue = DAPI. Images: $0.125 \mathrm{mg} / \mathrm{kg}$ Texas Red oligo without (A) and with (C) actin stain, and untreated mouse livers without (B) and with (D) actin stain. Scale bar $=100 \mu \mathrm{m}$. 

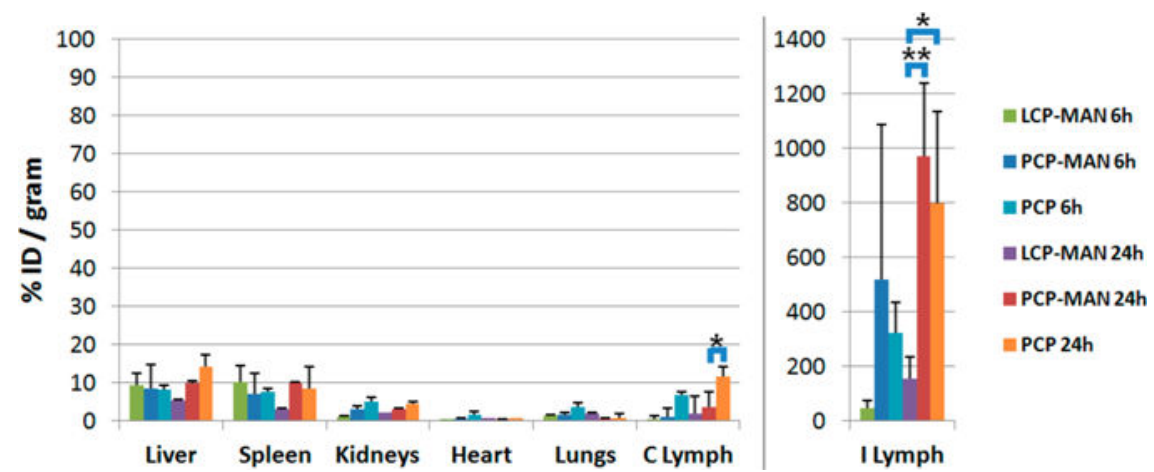

Figure 4.

LCP biodistribution upon subcutaneous administration. LCP particles encapsulating trace ${ }^{177} \mathrm{Lu}$ were coated with either $100 \mathrm{~mol} \%$ (PCP) or $20 \mathrm{~mol} \%$ (LCP) DSPE-PEG2k and administered subcutaneously to BALB/c mice in $5 \%$ glucose $(n=3)$. Major organs were harvested at various time points, and nanoparticle accumulation was determined radiometrically. $\mathrm{C}=$ contralateral; $\mathrm{I}=$ ipsilateral (local to injection site). $\mathrm{MAN}=$ mannose targeting. $* p<0.05 ; * p<0.01$. 

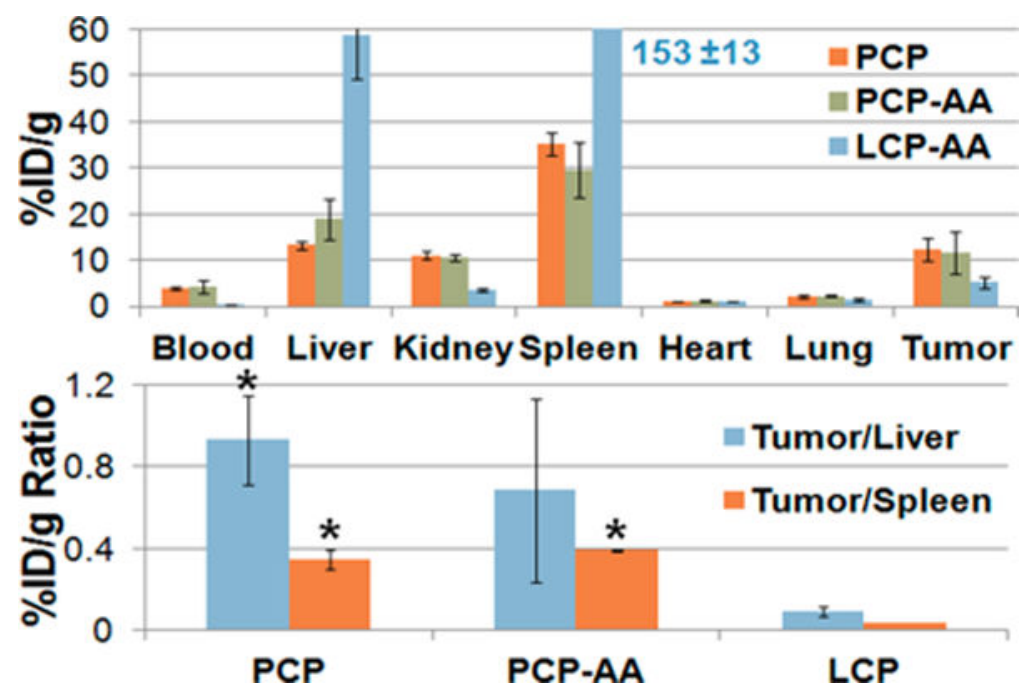

Figure 5.

LCP biodistribution in tumor-bearing mice. BALB/c mice were inoculated orthotopically with 4T1 tumors, and biodistribution of fully-PEGylated (PCP) and traditional (LCP) formulations containing trace ${ }^{177} \mathrm{Lu}$ was determined at $24 \mathrm{~h}$ post-tail vein injection. Average tumor size $=100 \mathrm{~mm}^{3}(n=3-4)$. ${ }^{*} p<0.02$ vs LCP-AA. 


\section{Table 1}

\section{Determination of PEG Density on LCP Liposomes ${ }^{a}$}

\begin{tabular}{llll}
\hline CaP cores per batch $(\# / \mathrm{mL}$ oil phase) & $10^{14}$ & PC headgroup CSA $\left(\mathrm{nm}^{2}\right)$ & 0.8 \\
core density (theor.) $\left(\mathrm{mg} / \mathrm{mm}^{3}\right)$ & 1.6 & PE headgroup CSA $\left(\mathrm{nm}^{2}\right)$ & 0.8 \\
core DOPA recovery $\left(\mathrm{mol}_{\text {out }} / \mathrm{mol}_{\text {in }}\right)$ & $35 \%$ & PEG chains $/ \mathrm{nm}^{2}$ (lipid bilayer) & 1.3 \\
PA headgroup CSA $\left(\mathrm{nm}^{2}\right)$ & 0.6 & PEG chains $/ \mathrm{nm}^{2}$ (PEG corona, est.) & 0.2 \\
\hline
\end{tabular}

${ }^{a}$ LCP nanoparticles comprising cores encapsulating ${ }^{177} \mathrm{Lu}$, coated with either 20 or $100 \mathrm{~mol} \%$ DSPE-PEG2k, and incorporating trace amounts of NBD-OPA, NBD-OPC, or DSPE-PEG-FITC were purified via sucrose gradient centrifugation and quantified via established fluorescence and radiometric methods. 